\title{
Invasive mechanical ventilation at 36 weeks post-menstrual age, adverse outcomes with a comparison of recent definitions of bronchopulmonary dysplasia
}

\author{
Milenka Cuevas Guaman $\mathbb{1}^{1} \cdot$ Nikou Pishevar $^{2} \cdot$ Steven H. Abman ${ }^{3} \cdot{\text { Martin Keszler } \mathbb{D}^{4} \cdot \text { William E. Truog }}^{5}$ • \\ Howard Panitch ${ }^{6}$ Leif D. Nelin ${ }^{2,7}$
}

Received: 14 October 2020 / Revised: 23 April 2021 / Accepted: 10 May 2021 / Published online: 25 May 2021

(c) The Author(s) 2021. This article is published with open access

\begin{abstract}
Objectives To determine whether the need for invasive mechanical ventilation (iMV) at 36 weeks PMA in patients with severe bronchopulmonary dysplasia (SBPD) identifies those patients at highest risk for tracheostomy or gastrostomy, and to compare sBPD with recent definitions of BPD.

Study design Observational study from Jan 2015 to Sept 2019 using data from the BPD Collaborative Registry.

Results Five hundred and sixty-four patients with sBPD of whom $24 \%$ were on iMV at 36 weeks PMA. Those on iMV had significantly $(p<0.0001)$ increased risk for tracheostomy or gastrostomy. The overall mortality rate was $3 \%$ and the risk for mortality was substantially greater in those on iMV than in those on noninvasive support at 36 weeks PMA (RR 13.8, 95\% CI $4.3-44.5, p<0.0001)$. When applying the NICHD definition (2016) $44 \%$ had Grade III BPD. When applying the NRN definition, 6\% had Grade 1 BPD, 70\% had Grade 2 BPD, and 24\% had Grade 3 BPD.

Conclusions Patients with SBPD who were on iMV at 36 weeks had a significantly greater risk of inhospital mortality and survivors had a significantly greater risk of undergoing tracheostomy and/or gastrostomy. The use of type 2 sBPD or Grade 3 BPD would enhance the ability to target future studies to those infants with SBPD at the highest risk of adverse long-term outcomes.
\end{abstract}

Milenka Cuevas Guaman

mxcuevas@texaschildrens.org

1 Neonatal/Perinatal Medicine, Baylor College of Medicine, Houston, TX, USA

2 Department of Pediatrics, The Ohio State University, Columbus, $\mathrm{OH}$, USA

3 Department of Pediatrics, University of Colorado, Denver, CO, USA

4 Department of Pediatrics, Brown University, Providence, RI, USA

5 Pediatrics, Children's Mercy-Kansas City, The University of Missouri-Kansas City School of Medicine, Kansas City, MO, USA

6 Department of Pediatrics, The Children's Hospital of Philadelphia, University of Pennsylvania, Philadelphia, PA, USA

7 Comprehensive Center for BPD, Nationwide Children's Hospital Columbus, Columbus, $\mathrm{OH}$, USA

\section{Introduction}

Despite marked improvements in the survival of extremely low birth weight preterm infants, bronchopulmonary dysplasia (BPD) remains the most common cause of morbidity and mortality in this population. In 2000, an NICHD workshop proposed a consensus definition of BPD that has become the most commonly used definition of BPD in the literature for the past 20 years. Using the 2000 consensus definition, severe BPD (sBPD) is defined as the need for positive pressure respiratory support or $\geq 30 \%$ oxygen at 36 weeks post-menstrual age (PMA) [1, 2]. However, many controversies persist regarding definitions of BPD and how to classify disease severity, which have resulted in new proposed definitions [3-5]. Definitions of BPD are especially important for developing standards of care, identifying disease mechanisms, anticipating childhood outcomes, and for designing rational clinical trials for the development of novel therapies [6-9]. Furthermore, the designation of sBPD from the 2000 NICHD workshop encompasses a very wide spectrum of disease severity; including infants requiring low-flow nasal cannula 
supplemental oxygen at an effective $\mathrm{FiO}_{2}$ of 0.30 to infants remaining dependent on invasive mechanical ventilation (iMV) at 36 weeks PMA [1, 2]. Recent studies have suggested that those infants who require higher levels of respiratory support, are especially vulnerable for having adverse outcomes including decreased survival, concurrent neurodevelopmental delays, and other co-morbidities $[3,10,11]$. However, data that more precisely identifies a specific subgroup of SBPD infants at the highest risk of adverse outcomes is lacking.

As part of the BPD Collaborative, we suggested dividing SBPD into two sub-catergories based on the respiratory support that patients were receiving at 36 weeks PMA, where type $1 \mathrm{sBPD}$ would include those patients on nasal cannula or noninvasive positive pressure support (i.e., high flow nasal cannula (HFNC), nasal continuous positive airway pressure (nCPAP), noninvasive intermittent positive pressure ventilation (nIPPV)) and type 2 sBPD would include those infants receiving iMV [12]. We based these 2 sub-categories of sBPD on the postulate that patients with type 2 sBPD would have greater mortality and morbidity than patients with type $1 \mathrm{sBPD}$. The variables in this study to evaluate morbidity were defined as the need for tracheostomy or gastrostomy, need for respiratory medications during NICU stay and at discharge, repiratiory support at discharge, length of stay (LOS), total ventilator days, and growth velocity ((discharge weight - birth weight)/LOS). Thus, the objective of this study was to determine if patients with SBPD who are receiving iMV at 36 weeks are more likely to suffer adverse outcomes. The BPD Collaborative has recognized the need for a more definite method of identifying disease severity among those patients currently categorized as SBPD [12]. The BPD Collaborative consists of sites interested in the care and outcomes of SBPD and who have established interdisciplinary care programs for the management of patients with sBPD. For this study we used data from the BPD Collaborative Registry. We also examined how the BPD Collaborative modification of the 2000 NIH Consensus Statement definition of sBPD compared with two recently proposed definitions of BPD using this cohort $[4,5]$.

\section{Materials/subjects and methods}

\section{Data sources and study sample}

There were six centers in the BPD Collaborative that had entered data into the inpatient data registry at the time of this data pull. The hospital are: Children's Mercy Hospital, Joe DiMaggio Children's Hospital, Johns Hopkins Hospital, Nationwide Children's Hospital, Vanderbilt University Medical Center and Women and Infants Hospital of Rhode
Island. Each institution contributing data to the registry obtained local IRB approval with waiver of consent. Each institution contributing data also entered into a Data Use Agreement with Nationwide Children's Hospital where the Registry is housed. The BPD Collaborative Data Registry collects a standardized dataset on all patients born at $<32$ weeks are entered into the data registry at 36 weeks PMA and were cared for by the dedicated BPD service at their respective institutions. The study was performed in accordance with the Declaration of Helsinki.

The 2000 NIH consensus definition of BPD was used to identify patients with SBPD, and included patients born at $<32$ weeks gestational age (GA) who received supplemental oxygen for 28 days, and were on $\geq 30 \%$ effective $\mathrm{FiO}_{2}$ or positive pressure at 36 weeks PMA [1]. Positive pressure was defined as HFNC, nCPAP, or nIPPV. iMV was defined as receiving IPPV via an endotracheal tube or a tracheostomy. Tracheostomy collar signifies supplemental oxygen delivery to the tracheostomized patient without mechanical ventilation, CPAP/PS (continuous positive airway pressure/ pressure support), or CPAP.The data collection included GA and growth parameters at birth and on the day of discharge. Growth velocity was defined as [(discharge weight - birth weight)/LOS]. Respiratory support data $\left(\mathrm{FiO}_{2}\right.$ and means of support) were collected at DOL 7, 14, 28 , and then at 36 weeks PMA and at discharge along with total ventilator days was collected as well. Data on the use of respiratory medications during the NICU stay and at discharge, such as diuretics, bronchodilators, systemic and inhaled steroids, were collected. Placements of tracheostomies and/or gastrostomies during the hospital stay were recorded. Respiratory support at discharge, classified as follows: none; low-flow nasal cannula (LFNC); tracheal collar; or positive pressure (i.e., HFNC, nCPAP, nIPPV, or IPPV) were recorded.

We also examined how the BPD Collaborative modification of the 2000 NIH Consensus Statement definition of SBPD compared with recently proposed definitions of BPD including the 2016 NICHD workshop definition [5] and the Neonatal Research Network (NRN) definition [4]. The NICHD consensus conference 2016 definition [5] suggested defining BPD in three grades in premature infants born at $<32$ weeks GA at 36 weeks PMA; where Grade I includes infants on nCPAP, nIPPV, or HFNC $(\geq 3 \mathrm{~L} / \mathrm{min})$ at $21 \% \mathrm{O}_{2}$ or nasal cannula 1 to $<3 \mathrm{lpm}$ or hood $\mathrm{O}_{2}$ at $22-29 \%$, or nasal cannula $<1 \mathrm{~L} / \mathrm{min}$ at $22-70 \% \mathrm{O}_{2}$; Grade II includes invasive IPPV at $21 \% \mathrm{O}_{2}, \mathrm{nCPAP}$, nIPPV, or HFNC at 22-29\% $\mathrm{O}_{2}$, cannula 1 to $<3 \mathrm{~L} / \mathrm{min}$ or hood $\mathrm{O}_{2}$ at $\geq 30 \% \mathrm{O}_{2}$, and nasal cannula $<1 \mathrm{~L} / \mathrm{min}$ at $>70 \% \mathrm{O}_{2}$; and Grade III includes invasive IPPV $>21 \% \mathrm{O}_{2}$, and nCPAP, nIPPV, or HFNC at $\geq 30 \%$. The NICHD 2016 consensus definition also included a Grade III(A) which was defined as patients whose early death (between 14 days postnatal age up until 
Table 1 Clinical characteristics of sBPD infants in this cohort.

\begin{tabular}{|c|c|c|c|}
\hline & Type $1 \mathrm{sBPD}$ & Type 2 sBPD & $p$ value \\
\hline \multicolumn{4}{|c|}{36 Week PMA respiratory support } \\
\hline Number & $429(76 \%)$ & $135(24 \%)$ & N/A \\
\hline Birth weight $(\mathrm{g})$ & $784(631-970)$ & $670(560-831)$ & $<0.0001$ \\
\hline Gestational age (weeks) & $26(24-27)$ & $25(24-26)$ & 0.003 \\
\hline Total ventilator days & $24(7-46)$ & $122(69-197)$ & $<0.0001$ \\
\hline Inhospital mortality & $0.7 \%$ & $9.9 \%$ & $<0.0001$ \\
\hline \multicolumn{4}{|c|}{ Medications during NICU stay } \\
\hline Diuretics & $49 \%$ & $78 \%$ & $<0.0001$ \\
\hline$\beta$-agonists & $39 \%$ & $72 \%$ & $<0.0001$ \\
\hline Inhaled corticosteroids & $46 \%$ & $70 \%$ & $<0.0001$ \\
\hline Systemic corticosteroids & $20 \%$ & $47 \%$ & $<0.0001$ \\
\hline \multicolumn{4}{|l|}{ Medications at discharge } \\
\hline Diuretics & $5.5 \%$ & $14 \%$ & 0.02 \\
\hline$\beta$-agonists & $36 \%$ & $56 \%$ & $<0.0001$ \\
\hline Inhaled corticosteroids & $38 \%$ & $60 \%$ & $<0.0001$ \\
\hline Systemic corticosteroids & $1.4 \%$ & $9.7 \%$ & $<0.0001$ \\
\hline \multicolumn{4}{|c|}{ Procedures during NICU stay } \\
\hline Tracheostomy & $3.5 \%$ & $23 \%$ & $<0.0001$ \\
\hline Gastrostomy tube & $21 \%$ & $45 \%$ & $<0.0001$ \\
\hline
\end{tabular}

Continuous data are shown as median (IQR), while categorical data are shown as percentages. The $p$ values were calculated using the Fisher's Exact Test.

36 weeks) was due to parenchymal lung disease and not associated with any other neonatal co-morbidities [5]. However, we only entered patients into the registry at diagnosis of sBPD at 36 weeks PMA so we were unable to assess earlier deaths. The mortality rate for this cohort was $3 \%$ therefore no secondary analysis was done on factors affecting mortality due to the low mortality rate with mortality defined as death prior to discharge with no cutoff for LOS. The NRN definition of BPD [4] was based solely on respiratory support at 36 weeks and included Grade 1 receiving nasal cannula $\leq 2 \mathrm{~L} / \mathrm{min}$; Grade II recieving HFNC, nCPAP, or NIPPV; and Grade 3 receiving invasive IPPV.

\section{Statistical analysis}

The continuous data in this cohort were nonparametric therefore the median and interquartile ranges are shown. The categorical data are shown as number and/or percent. For the nonparametric data comparisons between groups were made using a Mann-Whitney $U$ Test or where more than two groups were compared Kruskal-Wallis One Way Analysis of Variance on Ranks was used with a Student Newman-Keuls post hoc test to identify differences between groups. For the categorical data a two-tailed Fisher's Exact Test was used. Sigmaplot 12.0 (Sigma Scientific, Carlsbad, CA) was used for all statistical tests. A $p$ value $<0.05$ was considered to denote statistically significant.
Table 2 Relative risk for adverse outcomes in infants on iMV at 36 weeks PMA.

\begin{tabular}{lrll}
\hline Variable & RR & $95 \%$ CI & $p$ value \\
\hline Mortality & 13.8 & $4.3-44.5$ & $<0.0001$ \\
Tracheostomy & 6.6 & $3.7-11.5$ & $<0.0001$ \\
Gastrostomy tube & 2.1 & $1.6-2.8$ & $<0.0001$ \\
\hline
\end{tabular}

The $p$ values were calculated using the Fisher's Exact Test.

\section{Results}

There were 584 patients identified in the Registry with complete data at 36 weeks PMA, of which 20 had moderate BPD by the 2000 NIH consensus definition and were excluded. Of the 564 remaining patients with sBPD by the $2000 \mathrm{NIH}$ consensus definition, 135 (24\%) were receiving iMV at 36 weeks PMA. Patients on iMV at 36 weeks PMA weighed less at birth and were born earlier than those sBPD patients on noninvasive support at 36 weeks PMA (Table 1). Patients on iMV at 36 weeks PMA had more total ventilator days, longer LOS, and were treated more often with diuretics, $\beta$-agonists, or corticosteroids during their hospitalization than were sBPD patients on noninvasive support at 36 weeks PMA (Tables 1, 3).

The overall inhospital mortality rate after 36 weeks PMA for the entire cohort was 3\% (16/564). The risk for mortality after 36 weeks PMA was substantially greater in patients receiving $\mathrm{iMV}$ at 36 weeks PMA than in those SBPD patients on noninvasive respiratory support at 36 weeks PMA (RR 13.8, 95\% CI 4.3-44.5, $p<0.0001$ ). The risk for tracheostomy (RR 6.6, 95\% CI 3.7-11.5, $p<0.0001$ ) or gastrostomy (RR 2.1, 95\% CI 1.6-2.8, $p<0.0001$ ) were significantly greater for patients receiving iMV at 36 weeks PMA than compared to those who were receiving noninvasive respiratory support at 36 weeks PMA (Table 2). The sBPD patients on iMV at 36 weeks PMA had significantly slower growth velocity than did those on noninvasive support at 36 weeks PMA (Fig. 1).

Fewer sBPD patients who were on iMV at 36 weeks PMA were discharged on low-flow nasal cannula oxygen compared to sBPD patients on noninvasive respiratory support (Fig. 2). More sBPD patients who were on iMV at 36 weeks PMA were discharged on tracheal collar or positive pressure compared to sBPD patients on noninvasive support at 36 weeks PMA (Fig. 2). The sBPD patients on iMV at 36 weeks PMA were treated more often with $\beta$-agonists and corticosteroids at discharge compared to those on noninvasive respiratory support (Table 1).

When applying the NICHD workshop definition from 2016 to our cohort only $94 \%$ of the cohort could be classified, and of those that were classifiable $44 \%$ had Grade III BPD (Table 3). When applying the NRN definition [10], 


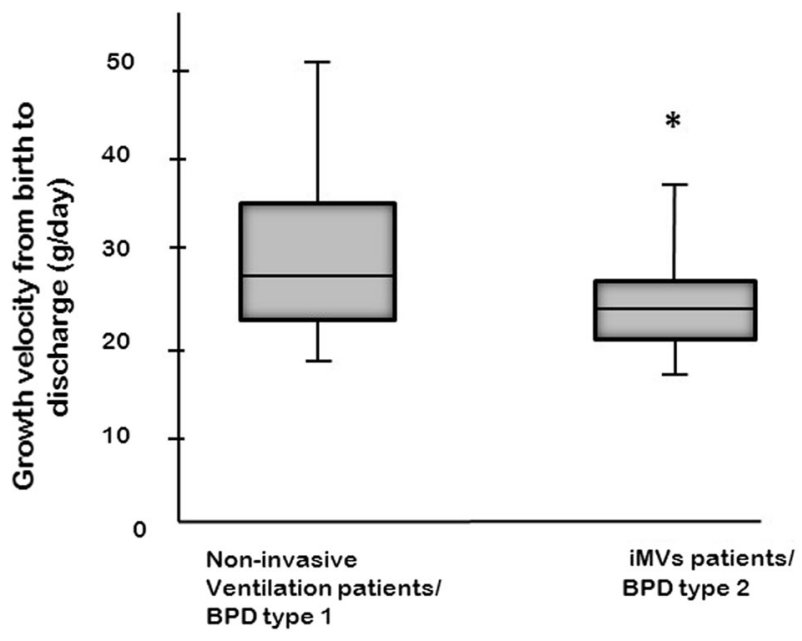

Fig. 1 Growth velocity distribution. Growth velocity defined as (discharge weight - birth weight/LOS) is lower in type 2 sBPD than type 1 sBPD. Asterisk indicates (*) type 2 sBPD different from type $1 \mathrm{sBPD}, p<0.001$. The $p$ values were calculated using a Mann-Whitney $U$ Test. Invasive mechanical ventilation (iMV).

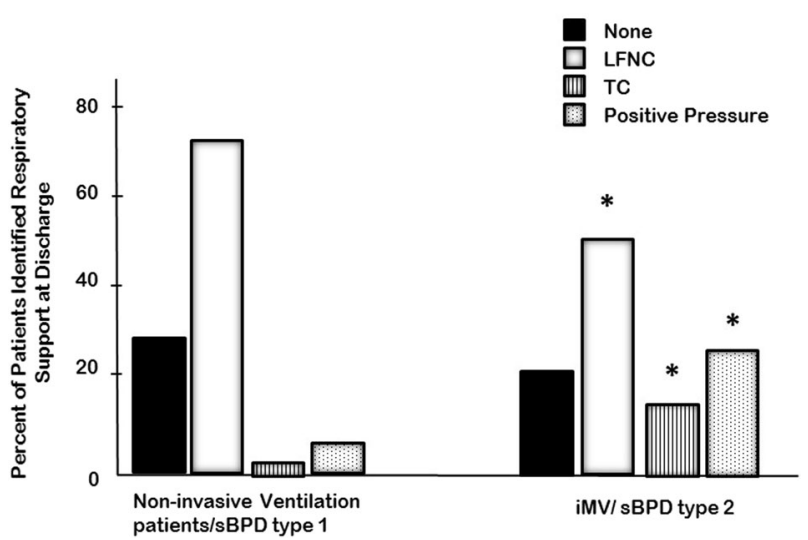

Fig. 2 Respiratory support at discharge. Respiratory support at discharge, classified as none; low-flow nasal cannula (LFNC), tracheostomy collar (TC) and positive pressure (i.e., HFNC. nCPAP, NIPPV, or IPPV). Asterisk indicates (*) type 2 sBPD different from type 1 sBPD, $p<0.001$. The $p$ values were calculated using Kruskal-Wallis One Way Analysis of Variance on Ranks with a Student Newman-Keuls post hoc test to identify differences between groups. Invasive mechanical ventilation (iMV), high flow nasal cannula (HFNC), nasal Continuous Positive Airway Pressure (nCPAP), noninvasive intermittent positive pressure ventilation (nIPPV), or intermittent positive pressure ventilation. Tracheostomy collar (TC) signifies supplemental oxygen delivery to the tracheostomized patient without mechanical ventilation, continuous positive airway pressure/ pressure support (CPAP/PS), or CPAP.

$100 \%$ of the cohort was able to be classified, and of those $6 \%$ had Grade 1 BPD, $70 \%$ had Grade 2 BPD and 24\% had Grade 3 BPD (Table 3). It should be noted that SBPD type 2 and Grade 3 BPD are defined by the same criteria at 36 weeks PMA. Mortality and LOS for the various definitions and severity classifications are shown in Table 3. Only the NICHD definition had patients that could not be classified, and this was $6 \%$ of the cohort, and the unclassifiable patients had a mortality of $10 \%$ and the longest LOS. The NRN definition placed $6 \%$ of patients classified as SBPD into Grade $1 \mathrm{BPD}$ and these patients had no mortality and the shortest LOS.

\section{Discussion}

BPD was originally described by Northway et al. [13]. Although changes in perinatal and neonatal care over time have improved survival of extremely preterm infants, the incidence of BPD continues to increase [14] and now includes a new phenotype that was characterized as an arrest of lung development by Jobe [15]. The evolving phenotypes of BPD are what lead to the NIH consensus definition in 2000 that included disease severity [1]. There has been growing recognition that the $2000 \mathrm{NIH}$ consensus definition of SBPD includes a very wide distribution of disease severity $[3,10,16,17]$. This wide distribution of disease severity makes it difficult for clinicians and researchers to determine what sBPD patients are actually at high-risk for mortality and morbidities. As a result, improving the outcomes of patients with SBPD, especially those who remain on iMV for prolonged periods of time, has been difficult within the constraints of the $2000 \mathrm{NIH}$ consensus definition of sBPD. Therefore, we sought to determine if patients with SBPD who remain ventilator dependent at 36 weeks PMA are at the highest risk for adverse outcomes. We chose to assess iMV at 36 weeks PMA because it is an easily identified and objective end-point. Reliance on the $\mathrm{FiO}_{2}$ without strict national guidelines for how to wean $\mathrm{FiO}_{2}$ results in subjectivity in the definition of BPD and/or the severity grading of BPD. Our findings support the hypothesis that patients who continue to receive iMV at 36 weeks PMA have the greatest risk of mortality and morbidities. Thus, the modification of the 2000 consensus definition of sBPD that we suggested in 2017 with the addition of type 1 and type 2 sBPD does provide a more precise identification of the highest risk infants. We should point out that the NRN definition of Grade 3 BPD also identifies the patients in this cohort with the greatest risk of adverse outcomes. Thus, the use of Grade 3 BPD identifies patients at the highest risk for adverse outcomes and will be useful for future epidemiologic studies and randomized clinical trials.

The lack of consensus in approach to patients with sBPD continues to hamper the development of new methods or therapies to improve outcomes [18, 19]. Large multicenter interventional trials are desperately needed for this patient population, but the lack of consensus introduces the concept of "equipoise", which then often becomes a reason not to perform the study. Furthermore, the wide spectrum of 
Table 3 Comparison of definitions applied to this cohort.

\begin{tabular}{llcr}
\hline & Distribution & Mortality & LOS \\
\hline $\begin{array}{l}\text { BPD Collaborative modification of sBPD } \\
\quad\end{array}$ & $76 \%$ & & \\
$\quad$ Type 1 & $24 \%$ & $0.7 \%$ & $110(87-142)$ \\
$\quad$ Type 2 & & $9.9 \%$ & $190(131-309)$ \\
NICHD 2016 & $26 \%$ & & \\
Grade I & $26 \%$ & $0 \%$ & $95(69-127)$ \\
Grade II & $42 \%$ & $0 \%$ & $116(91-149)$ \\
Grade III & $6 \%$ & $5.7 \%$ & $153(120-214)$ \\
$\quad$ Non-classifiable & & $10 \%$ & $234(122-376)$ \\
NRN 2019 & $6 \%$ & & \\
Grade 1 & $70 \%$ & $0 \%$ & $99(86-137)$ \\
Grade 2 & $24 \%$ & $9.9 \%$ & $115(90-146)$ \\
Grade 3 & & $9.9 \%$ & $190(131-309)$ \\
\hline
\end{tabular}

Distribution and Mortality data shown as percent. LOS (length of stay) data shown as median (IQR). disease currently classified as sBPD leads to potentially targeting a population that may not be expected to benefit from the therapy. An improved ability to identify the highest risk patients with sBPD will allow for the development and study of precision management strategies tailored specifically to this high-risk group. For example, the highest risk sBPD patients may be those most likely to benefit from pulmonary targeted pharmacotherapies. However, the widely variable phenotype currently lumped under sBPD likely hampers the recognition of meaningful clinical responses to common pharmacotherapies. Most of which are already utilized in this population with little or no evidence to support their use, such as $\beta$-agonists, diuretics, and inhaled corticosteroids. It has been found that the utilization of these pharmacotherapies vary widely between centers $[18,19]$. In a recent retrospective cohort study using the Pediatric Health Information System database, Bamat et al. [19] concluded that infants with SBPD are exposed to an alarming number of medications of unclear efficacy and safety, with marked variation between centers.

We have utilized the terms type 1 and type 2 sBPD as a method of separating sBPD patients into two distinct potential phenotypes [12]. The definition of sBPD is based on the $2000 \mathrm{NIH}$ consensus definition [1]. However, it is clear 20 years later that the 2000 NIH Consensus definition no longer adequately meets the changing needs of neonatology $[10,20]$. In fact, there has been a very lively debate in the literature over the last several years regarding new definitions, what elements they should include, and what they should be able to prognosticate [20-27]. We demonstrate in this study that those BPD patients on iMV at 36 weeks PMA are at the highest risk for inhospital mortality and morbidities. The 2016 NICHD definition of BPD does include newer modes of noninvasive ventilation that weren't available in 2000 , although the definition proposed is based on a very complex interaction between $\mathrm{FiO}_{2}$ and respiratory support at 36 weeks PMA [5]. Interestingly, in our cohort $6 \%$ of patients were not able to be classified using the 2016 NICHD definition due to lack of reliable $\mathrm{FiO}_{2}$ data at 36 weeks PMA. In 2019 the NRN also proposed a series of definitions of BPD based solely on the level of respiratory support needed at 36 weeks PMA and tested the various definitions to find the one that "best" predicted death or serious respiratory morbidity through 18-26 months corrected age using the area under the receiver operator characteristic curve (AUROC) [4]. They found that defining Grade 1 as low-flow nasal cannula, Grade 2 as noninvasive positive pressure, and Grade 3 as iMV gave the best predictive value in terms of the AUROC for late death or serious respiratory morbidity [10]. Although, it should be pointed out that the AUROC was only 0.79 , suggesting that either newer definitions and/or developing phenotypic clusters within current definitions are needed to improve the ability to develop a classification scheme that identifies those at the very highest risk for later adverse pulmonary outcomes. The 2019 NRN definition is the easiest to assign since it only requires one timepoint and does not use supplemental oxygen [4]. When the NRN criteria was applied to our cohort, we also found that $6 \%$ of our sBPD patients were defined as having Grade 1 BPD, and that the patients identified as Grade 1 BPD had no inhospital mortality and a short LOS. Thus, the NRN criteria may provide a more precise classification compared to our suggested sub-grouping of sBPD since it includes both lower and higher severity of disease.

There are some limitations to our study that should be considered. First, the cohort only consisted of patients with established SBPD as this was our population of interest for this study. However, this may limit the generalizability of our findings to all preterm infants classified as having BPD. 
Second, it is possible that there is referral bias in our cohort, since the six centers contributing data to this cohort are all centers that have an established BPD program and therefore may not be representative of the entire population of SBPD patients. Finally, our dataset was limited to inhospital mortality and morbidity. Going forward the BPD Collaborative is developing and implementing an outpatient dataset, which will be useful in determining the effect of invasive IPPV at 36 weeks on long-term outcomes.

In conclusion, we found that in a cohort of patients with sBPD, those that were receiving iMV at 36 weeks PMA were at the highest risk of inhospital mortality and morbidity. Patients with SBPD who were on iMV at 36 weeks had a significantly greater risk of inhospital mortality and survivors had a significantly greater risk of undergoing tracheostomy and/or gastrostomy placement prior to discharge. Our study adds support to the growing perception that new definitions and/or classifications of BPD are needed going forward to better capture the breadth of phenotypes and endotypes that characterize this chronic lung disease of prematurity. We suggest that future definitions include objective methods for differentiating the wide spectrum of disease presentation and progression representing the severest forms of BPD. Throughout this study, it has been shown that the BPD Collaborative definition of type 2 sBPD and the NRN definition of Grade 3 BPD are the same diagnosis since it specifies a sub-category of patients who have iMV at 36 weeks PMA, however the NRN definition also categorizes a subset of sBPD infants into a less severe category as well in which those infants had no mortality and a shorter LOS. As a start, the utility of using the NRN definition for Grade 3 BPD would be most feasible given the singular timepoint to make the diagnosis and this would enhance the ability to target future studies to those infants with SBPD at the highest risk of adverse long-term outcomes.

\section{Data availability}

The BPD Collaborative Data Registry collects a standardized dataset on all patients born at $<32$ weeks who were cared for by the dedicated BPD service at their respective institutions.

Acknowledgements Publication costs were generously supported by Texas Children's Hospital Young Investigators Endowed Fund.

Author contributions All authors approved the final paper as submitted and agree to be accountable for all aspects of the work. MCG and LDN conceptualized and designed the study, drafted the initial paper, coordinated and supervised data analisys and reviewed and revised the paper. NP carried out the initial analyses, and reviewed and revised the paper. MK, WET, and HP helped conceptualize and design the study, and critically reviewed the paper for important intellectual content.

\section{Compliance with ethical standards}

Conflict of interest The authors declare no competing interests.

Ethical approval The study was performed in accordance with the Declaration of Helsinki.

Publisher's note Springer Nature remains neutral with regard to jurisdictional claims in published maps and institutional affiliations.

Open Access This article is licensed under a Creative Commons Attribution 4.0 International License, which permits use, sharing, adaptation, distribution and reproduction in any medium or format, as long as you give appropriate credit to the original author(s) and the source, provide a link to the Creative Commons license, and indicate if changes were made. The images or other third party material in this article are included in the article's Creative Commons license, unless indicated otherwise in a credit line to the material. If material is not included in the article's Creative Commons license and your intended use is not permitted by statutory regulation or exceeds the permitted use, you will need to obtain permission directly from the copyright holder. To view a copy of this license, visit http://creativecommons. org/licenses/by/4.0/.

\section{References}

1. Jobe AH, Bancalari E. Bronchopulmonary dysplasia. Am J Respir Crit Care Med. 2001;163:1723-9.

2. Ehrenkranz RA, Walsh MC, Vohr BR, Jobe AH, Wright LL, Fanaroff AA, et al. Validation of the National Institutes of Health consensus definition of bronchopulmonary dysplasia. Pediatrics. 2005;116:1353-60.

3. Isayama T, Lee SK, Yang J, Lee D, Daspal S, Dunn M, et al. Revisiting the definition of bronchopulmonary dysplasia: effect of changing panoply of respiratory support for preterm neonates. JAMA Pediatr. 2017;171:271-9.

4. Jensen EA, Dysart K, Gantz MG, McDonald S, Bamat NA, Keszler M, et al. The diagnosis of bronchopulmonary dysplasia in very preterm infants. an evidence-based approach. Am J Respir Crit Care Med. 2019;200:751-9.

5. Higgins RD, Jobe AH, Koso-Thomas M, Bancalari E, Viscardi RM, Hartert TV, et al. Bronchopulmonary dysplasia: executive summary of a workshop. J Pediatr. 2018;197:300-8.

6. Islam JY, Keller RL, Aschner JL, Hartert TV, Moore PE. Understanding the short- and long-term respiratory outcomes of prematurity and bronchopulmonary dysplasia. Am J Respir Crit Care Med. 2015;192:134-56.

7. Hacking DF, Gibson AM, Robertson C, Doyle LW. Respiratory function at age 8-9 after extremely low birthweight or preterm birth in Victoria in 1997. Pediatr Pulmonol. 2013;48:449-55.

8. Sillers L, Alexiou S, Jensen EA. Lifelong pulmonary sequelae of bronchopulmonary dysplasia. Curr Opin Pediatr. 2020;32:252-60.

9. Ryan RM. A new look at bronchopulmonary dysplasia classification. J Perinatol. 2006;26:207-9.

10. Poindexter BB, Feng R, Schmidt B, Aschner JL, Ballard RA, Hamvas A, et al. Comparisons and limitations of current definitions of bronchopulmonary dysplasia for the prematurity and respiratory outcomes program. Ann Am Thorac Soc. 2015;12:1822-30.

11. Padula MA, Grover TR, Brozanski B, Zaniletti I, Nelin LD, Asselin JM, et al. Therapeutic interventions and short-term outcomes for infants with severe bronchopulmonary dysplasia born at $<32$ weeks' gestation. J Perinatol. 2013;33:877-81. 
12. Abman SH, Collaco JM, Shepherd EG, Keszler M, CuevasGuaman M, Welty SE, et al. Interdisciplinary care of children with severe bronchopulmonary dysplasia. J Pediatr. 2017;181:12-28. e11.

13. Northway WH Jr., Rosan RC, Porter DY. Pulmonary disease following respirator therapy of hyaline-membrane disease. Bronchopulmonary dysplasia. N Engl J Med. 1967;276:357-68.

14. Stoll BJ, Hansen NI, Bell EF, Walsh MC, Carlo WA, Shankaran S, et al. Trends in care practices, morbidity, and mortality of extremely preterm neonates, 1993-2012. JAMA. 2015;314:1039-51.

15. Jobe AH. The new bronchopulmonary dysplasia. Curr Opin Pediatr. 2011;23:167-72.

16. Hines D, Modi N, Lee SK, Isayama T, Sjors G, Gagliardi L, et al. Scoping review shows wide variation in the definitions of bronchopulmonary dysplasia in preterm infants and calls for a consensus. Acta Paediatr. 2017;106:366-74.

17. Thebaud B, Goss KN, Laughon M, Whitsett JA, Abman SH, Steinhorn RH, et al. Bronchopulmonary dysplasia. Nat Rev Dis Prim. 2019;5:78.

18. Guaman MC, Gien J, Baker CD, Zhang H, Austin ED, Collaco JM. Point prevalence, clinical characteristics, and treatment variation for infants with severe bronchopulmonary dysplasia. Am J Perinatol. 2015;32:960-7.

19. Bamat NA, Kirpalani H, Feudtner C, Jensen EA, Laughon MM, Zhang $\mathrm{H}$, et al. Medication use in infants with severe bronchopulmonary dysplasia admitted to United States children's hospitals. J Perinatol. 2019;39:1291-9.

20. Isayama T, Shah PS. Need for an International Consensus on the Definition of Bronchopulmonary Dysplasia. Am J Respir Crit Care Med. 2019;200:1323-4.

21. Jensen EA. Reply to Bancalari et al. and to Isayama and Shah. Am J Respir Crit Care Med. 2019;200:1324-5.

22. Bancalari E, Claure N, Jain D. Diagnostic classification of bronchopulmonary dysplasia: a compromise between defining lung disease versus long-term outcome prediction. Am J Respir Crit Care Med. 2019;200:1322-3.

23. Stoecklin B, Simpson SJ, Pillow JJ. Bronchopulmonary dysplasia: rationale for a pathophysiological rather than treatment based approach to diagnosis. Paediatr Respir Rev. 2019;32:91-7.

24. Jensen EA, Wright CJ. Bronchopulmonary dysplasia: the ongoing search for one definition to rule them all. $\mathrm{J}$ Pediatr. 2018;197:8-10.

25. Jobe AH, Bancalari EH. Controversies about the definition of bronchopulmonary dysplasia at 50 years. Acta Paediatr. 2017;106:692-3.

26. Meyer S, Franz AR, Bay J, Gortner L. Developing a better and practical definition of bronchopulmonary dysplasia. Acta Paediatr. 2017;106:842.

27. Bancalari E, Jain D. Bronchopulmonary dysplasia: can we agree on a definition? Am J Perinatol. 2018;35:537-40. 